\title{
Soziale Arbeit in Geflüchtetenunterkünften
}

\section{Menschenrechte unter Verschluss}

Geflüchtete Familien und alleinstehende Erwachsene leben nach ihrer Einreise in Deutschland in allen Bundesländern zunächst in einer Landeserstaufnahmeeinrichtung. Die Lebenswelten der Betreffenden sind durch die Asyl- und Ausländer_innengesetzgebung und Routinen Asyl verwaltender Organisationen bestimmt. Die Selbstbestimmung ist genauso eingeschränkt wie die gesellschaftliche Teilhabe: Der Zugang zu Gesundheitsversorgung, zum (Aus-)Bildungssystem und regulären Arbeitsmarkt ist durch ihren Ausländer_innenstatus nach dem Asylgesetz reglementiert.

I $\mathrm{n}$ den Unterkünften teilen sich die Menschen zusammen mit anderen ein Zimmer und nutzen Gemeinschaftsräume wie Küche oder Duschanlagen. Das Zusammenleben auf engem Raum, die Sorge um den Ausgang des Asylantrags und die Ungewissheit über die Zukunft begünstigen Konflikte (Bauer 2017). Hinzu kommen rassistische Übergriffe und Gewalt (BKA 2019). Auf die strukturell evozierten Problemlagen hat das Bundesfamilienministerium gemeinsam mit dem

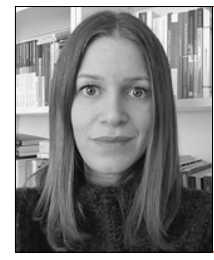

\section{Caroline Schmitt}

Johannes Gutenberg-Universität Mainz, Mainz, Deutschland *1984; Dr., Diplom-Pädagogin, wissenschaftliche Mitarbeiterin am Institut für Erziehungswissenschaft der Johannes GutenbergUniversität Mainz.

schmitt_caroline@gmx.de

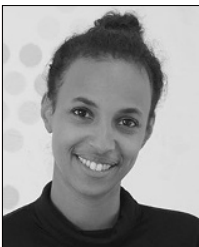

\section{Samia Aden}

Kassel, Deutschland

*1987; M. A., wissenschaftliche Mitarbeiterin im Fachgebiet Sozialisation mit dem Schwerpunkt Migration und interkulturelle Bildung der Universität Kassel.

s.aden@uni-kassel.de

Zusammenfassung Dieser Beitrag befasst sich mit Möglichkeiten und Begrenzungen sozialarbeiterischen Handelns in Geflüchtetenunterkünften in Deutschland. Er zeigt auf Basis einer Fallvignette Strategien einer pädagogischen Fachkraft auf, ihr pädagogisches Mandat auszufüllen, dabei aber an Grenzen des Machbaren und der eigenen Belastbarkeit zu stoßen. Ausgehend von diesem Fall reflektiert der Beitrag die Bedeutsamkeit einer menschenrechtsbasierten und interprofessionellen Verortung Sozialer Arbeit im Spannungsfeld von nationalstaatlichen Partikularinteressen und universalistischem Gleichheitsanspruch.

Schlüsselwörter Geflüchtetenunterkunft, Soziale Arbeit, Menschenrechte, Kinderrechte, Interprofessionalität
UN-Kinderhilfswerk im Jahr 2016 reagiert und die Bundesinitiative „Schutz von geflüchteten Menschen in Flüchtlingsunterkünften “ ins Leben gerufen. Ziel ist eine Verbesserung der Unterbringungssituation und Etablierung einheitlicher Standards (BMFSFJ/UNICEF 2018). Eine rechtsverbindliche Standardisierung von Schutzkonzepten gibt es derzeit jedoch nicht. Es sind vor allem zivilgesellschaftliche Initiativen, die über die Situation in den Unterkünften berichten und ihre Konformität mit Menschen- und Kinderrechten in Zweifel ziehen (Bayerische Flüchtlingsrat 2019).

\section{Soziale Arbeit im Spannungsfeld}

Pädagog_innen sind in Geflüchtetenunterkünften etwa in der Sozialberatung von dort ansässigen Trägern tätig, führen Bildungsprojekte durch oder unterstützen bei der Kinderbetreuung. Bisherige Studien zeichnen das Bild vielfach engagierter Fachkräfte, die sich jedoch durch das asylrechtlich gerahmte Setting in ihrer professionellen Handlungsfähigkeit bedroht sehen (Eichinger und Schäuble 2018). Im Berufsalltag führen drohende und sichtbar werdende Kindeswohlgefährdungen und Abschiebepraktiken zu der Frage, inwiefern pädagogisches Handeln unter diesen Bedingungen überhaupt möglich ist.

\section{Arbeitsort „Geflüchtetenunterkunft":} eine Pädagogin erzählt

In Anknüpfung an diese Problematik geben wir auf Basis einer Fallvignette Einblick in die Perspektive einer Pädagogin auf ihr Arbeitsfeld. Die Pädagogin ist Leiterin eines Horts auf dem Gelände einer Geflüchtetenunterkunft in Deutschland ${ }^{1}$. Die Vignette basiert auf Beobachtungsmaterial, einem qualitativen Interview und Auszügen aus dem pädagogischen Konzept 


\section{Extrablick: Soziale Arbeit als Menschenrechtsprofession}

des Horts. Das Material wurde zwischen Juni 2019 und März 2020 erhoben.

\section{Kinderrechte im Hort}

Marianne Rudolf ${ }^{2}$ erzählt im Interview und während unserer Beobachtungen ausgiebig von ihrer Arbeit. Ihre Erzählungen machen ein Spannungsfeld zwischen ihrem Arbeitsverständnis und den Rahmenbedingungen ihrer Tätigkeit sichtbar. Den Hort beschreibt sie als bedeutsame Anlaufstelle der Kinder in der Geflüchtetenunterkunft (Interview, Zeile 110-115). Hier treffen sie auf Gleichaltrige, haben Raum zum Spielen und Hausaufgabenmachen. Der Hort ist paritätisch mit Kindern aus der Stadt und Kindern aus der Unterkunft belegt und ermöglicht geflüchteten $\mathrm{Fa}$ milien eine Kontaktknüpfung über die Unterkunft hinaus (Interview, Zeile 1280). Im pädagogischen Konzept sind das „Recht auf Mitbestimmung “, „Recht auf freie Entfaltung“, der „Anspruch eines jeden Kindes auf kindgerechte Lebens- und Lernbedingungen“ und „verlässliche Beziehungen“ sowie das Recht auf eine „gleichberechtigte Teilhabe“ aller Kinder am Hortgeschehen herausgestellt. In Kinderkonferenzen haben Kinder die Möglichkeit, ihre Anliegen zum Ausdruck zu bringen, auch ein kindbezogenes Beschwerdemanagement ist eingerichtet. Im Zuge unserer Beobachtungen erfahren wir von einer von den Kindern selbst verwalteten Bibliothek und sehen, wie Kinder den Eingangsbereich in ihrem Sinne mit Bänken und Spielzeug gestalten (Protokoll vom 06.03.2020). Partizipation und Teilhabe erfahren durch die Lebenswirklichkeiten in der Unterkunft jedoch eine umfassende Beschneidung.

\section{Lebenswirklichkeit "Abschiebung”}

Frau Rudolf zeigt im Interview den Vollzug von Abschiebungen auf. Der pädagogische Anspruch bricht in sich zusammen:

wir wollen [...] dass die Kinder [...] sich unterstützt füblen und dann plötzlich und das Kind [...] hätte womöglich aufs Gymnasium wechseln können [...] die Familie ist weg. Wir wissen nicht wo [...] dann kommen die Kinder aus der Schule [...] erzäblen: Das Kind ist weg (Interview, Zeile 121-131).

Der erfolgreich verlaufende Bildungsweg eines Kindes endet abrupt mit der Abschiebung. Die Pädagogin erfährt hiervon erst durch die anderen Kinder im Hort, da Abschiebungen in der Nacht vollzogen werden:

zwischen vier und fünf Uhr nachts. Abschiebung bekommen wir nicht mehr mit, wir kriegen jetzt mit, dass die Kinder nicht mehr da sind [...]. Das ist schwer (Interview, Zeile 149-151).
Frau Rudolf bringt ihre Fassungslosigkeit zum Ausdruck. Eindrücklich stellt sie die erlebte Ohnmacht heraus, wenn Kinder mit ihren Familien von heute auf morgen aus ihrem Alltag gerissen werden und ihre alltäglich gebrauchten Dinge, die nicht mitgenommen werden konnten, zurückbleiben:

all die Schätze, die die Kinder mit nach Hause genommen haben, die durch Spenden ins Haus gekommen sind, liegen dann nachher auf der Wiese vom Schulbuch bis zum Teddybär [...] die Leute, die hier wohnen kommen und holen sich noch was sie brauchen können (Interview, Zeile 132-136).

Der auf der Wiese liegende Teddybär ist Ausdruck pädagogischer Bemühungen und zivilgesellschaftlicher Solidarität. Solidarität und Pädagogik scheinen an der Grenze ihrer Einflussmöglichkeiten angelangt zu sein.

\section{„Ein Altar für Mina": Trauerarbeit im Kinderhort}

Statt Spiel, Spaß und Bildung rückt eine Trauerarbeit mit den Kindern in den Fokus der Arbeit - eine Aufgabe, die im teilhabeorientierten Hortkonzept unerwähnt bleibt, aber Teil der pädagogischen Arbeit ist:

Vor zwei Jahren [...] haben ne Handvoll Kinder [...] einen Altar gemacht für Mina. [...]. Das Kind ist abgeschoben worden. [...] Die sind jeden Tag dahin, haben Briefchen [...] dabin gelegt. [...] wenn dann Besucher gekommen sind, Politiker und dann hab ich gesagt: dann zeig ich Ihnen jetzt mal noch die Gedenkstätte von Mina. [...] Die Kinder müssen ja irgendwie damit umgehen, wir können ibnen ja nicht helfen. Auch wenn die uns als Macher erleben [...] haben [wir] ja keine politische Macht (Interview, Zeile 121-165).

Die Interviewte stellt die Ambivalenz heraus, einerseits von den Kindern als Macherin wahrgenommen zu werden, sich gleichzeitig aber handlungsunfähig zu fühlen. Den von den Kindern errichteten Altar zeigt sie jenen, die über „politische Macht" verfügen und erschließt sich eine kleine Form der Einflussnahme. Ein Traueraltar, wie er im Gedenken an tote Menschen üblich ist, symbolisiert hier den Abbruch sozialer Beziehungen und die Beendigung eines aufgebauten Lebens in Deutschland. Für die Kinder und Pädagog_innen schafft er einen Ort zur Trauerbewältigung und Thematisierung eigener Ängste.

\section{"Schwer zu verkraften"}

Im Kontrast zur heutigen Arbeitssituation erlebte die Pädagogin ihr pädagogisches Wirken in der Vergangen- 
heit als weitreichend und konnte sich für Hort und Kinder einsetzen und Abschiebungen verhindern:

ich durfte auch immer stark agieren und das hat richtig gut getan, aber man kämpft sich müde [...] heute schaffe ich das nicht mehr. Also heute ist es auch gar nicht mehr möglich [...]. Damals [...] die Gesetze [...] haben noch Lücken gehabt in dem Rabmen [...] man [...] noch agieren konnte (Interview, Zeile 350-355).

Die Metapher des Kampfes („man kämpft“) hebt die Macht der unbesiegbar erscheinenden Gegner_innen hervor. Das pädagogische Konzept wird zwar in den Räumen des Horts umgesetzt; dieser erscheint aber als ständig bedrohte Insel.

\section{Bewältigungsherausforderung „Abschiebung”}

Die Fallvignette verdeutlicht, dass das Leben für die Kinder und ihre Familien in Geflüchtetenunterkünften mit Beschneidungen in der Selbstbestimmung und Ängsten einhergeht. Kommt es zur Abschiebung, münden die Familienbiografien im Ungewissen. Netzwerke und eingeschlagene Bildungswege brechen ab. Verabschiedungen von Spielpartner_innen und erwachsenen Bezugspersonen sind wegen nächtlicher Abschiebungen nicht möglich. Auch für die Zurückbleibenden gehen Abschiebungen mit hohen Bewältigungsherausforderungen einher. Kindern und Familien wird die Gefahr, plötzlich selbst „weg“ sein zu können, stets vor Augen gehalten. Pädagog_innen erleben die Diskrepanz zwischen pädagogischem Mandat und der Realität der Asylpolitik.

\section{Menschen- und Kinderrechte unter Verschluss}

Die im Rahmen der Fallvignette aufgezeigte Perspektive der Fachkraft fordert die Soziale Arbeit grundlegend heraus, Umgangsweisen mit dem diffizilen Handlungs- und Arbeitsfeld der Fluchtsozialarbeit zu finden und weder die betroffenen Familien und Kinder, noch einzelne pädagogische Fachkräfte allein zu lassen. Die im Hortkonzept formulierten Rechte von Kindern auf Selbstbestimmung und Rechte, wie sie als Teilbereich der Menschenrechte in der Kinderrechtskonvention der Vereinten Nationen formuliert sind - wie etwa das Recht auf soziale Sicherheit (Art. 26), angemessene Lebensbedingungen (Art. 27) oder das Recht auf Freizeit, Erholung und Beteiligung (Art. 31) - sind für Kinder, die von Abschiebung betroffen sind, nicht mehr gewährt. Exemplarisch macht die Fallvignette somit auf die Diskrepanz zwischen pädagogischem Mandat und den Lebensbedingungen in den Unterkünften und Abschiebungen aus Deutschland aufmerksam.

\section{Tripelmandat und pädagogisches Handeln}

Was bedeutet diese Diskrepanz nun für die Soziale Arbeit? Sozialarbeitende agieren im Kontext von Flucht und Asyl in einem besonders zugespitzten Konfliktfeld. Sie wollen die Betreffenden unterstützen, stoßen aber an Grenzen, da ihre Adressat_innen nicht im Besitz voller Bürger_innenrechte sind. Orientierung (Schäuble 2018, S. 93) kann die Soziale Arbeit jedoch in ihrem Selbstverständnis als Menschenrechtsprofession finden. Eine solche Perspektive geht u. a. auf Silvia Staub-Bernasconi (2019, S. 85-87) zurück, die das Doppelmandat Sozialer Arbeit, einerseits die Adressat_innen Sozialer Arbeit zu unterstützen und andererseits gesellschaftliche Ordnungsinteressen zu verfolgen, um eine professionsethische, menschenrechtliche Dimension erweitert hat. Menschen- und Kinderrechte fungieren in einem solchen Verständnis als Bezugsrahmen, um zu reflektieren, ob und wie sie in nationalstaatliches und internationales Recht, etwa die Asyl- und Ausländer_innenpolitik, übersetzt wurden. Aus dieser Perspektive heraus lassen sich Leerstellen identifizieren, kommunizieren und bearbeiten, an welchen Menschen- und Kinderrechte unberücksichtigt bleiben und pädagogische Ziele nur schwer zu realisieren sind.

\section{Menschenrechtsbildung und Professionsverständ-} nis

Das Tripelmandat verweist unmittelbar auf eine Bildungsaufgabe für die Soziale Arbeit: Basis ist zunächst, dass Sozialarbeiter_innen die Menschenrechte überhaupt kennen. Hierzu ist eine curricular institutionalisierte Menschenrechtsbildung in (Hoch-)Schulen und fortwährende Thematisierung in der Fort-, Aus- und Weiterbildung notwendig, die sich gegen Diskriminierung und für eine inklusive Gesellschaft ausspricht. Vor allem im Feld der internationalen Sozialen Arbeit ist ein solches Professions- und Disziplinverständnis vielfach herausgestellt worden (Healy 2008) und wird auch in Deutschland vertreten (Kleibl und Lutz 2020).

\section{Interprofessionalität}

Die menschenrechtliche Bezugnahme tangiert dabei nicht ausschließlich Pädagog_innen; sie verlangt nach interprofessionellen Netzwerken etwa mit Jurist_innen, Ärzt_innen und Psycholog_innen. Soziale Arbeit kann in interprofessionellen Netzwerken die Aufgabe übernehmen, mit professionsethischer Stimme und gemeinsam mit den Betreffenden befähigende Netzwerke aufzubauen, die die sozialarbeiterischen Handlungsmöglichkeiten sowie Möglichkeiten der Familien und Kinder erweitern. In diesem Sinne geht sie über eine Einzelfallhilfe hinaus und strebt grundlegende Änderun- 


\section{Extrablick: Soziale Arbeit als Menschenrechtsprofession}

gen wie etwa einen verbesserten Zugang geflüchteter Menschen zum Gesundheitswesen an (Schäuble 2018, S. 90). Sie fungiert als „Agentin des sozialen Wandels wie der persönlichen Befähigung “ (Röh 2013, S. 148) und verfolgt das Anliegen, „sowohl strukturelle Mängel zu thematisieren als auch persönliche Erziehungs-, Bildungs- und Entwicklungsprozesse zu begleiten, anzuregen und aktiv zu fördern“ (ebd.).

\section{Neue Allianzen und transnationale Öffnung}

Zivilgesellschaftliche Initiativen können für die Soziale Arbeit hierbei (nicht nur) im Bereich von Fluchtmigration wichtige Netzwerkpartner_innen sein, ohne dass sie sozialarbeiterische Aufgaben ersetzen. Sie verfügen über wertvolle Erfahrungen und Kooperationen - etwa zu unabhängigen Rechtsberatungsstellen - und initiieren selbst eigene Projekte, beispielsweise inklusive Wohnformen (Schiffauer et al. 2017). Furman et al. (2008) schlagen vor, soziale Dienste noch stärker als bisher mit zivilgesellschaftlichen Akteur_innen zu verzahnen - dies auch und vor allem über nationalstaatliche Grenzen hinweg, sodass etwa abgeschobene Familien zumindest in transnationale Hilfenetzwerke eingebunden werden. Transnationale wie lokale Kooperationen sind vor dem Hintergrund der transnationalen Lebenswelten der Familien besonders relevant und können bei der Erarbeitung von Lebensperspektiven unterstützen.

\section{Begrenzung von Bürger_innenrechten}

Gleichwohl wir damit auf Perspektiven für das Feld der Fluchtsozialarbeit hinweisen, ist es in hohem Maße eine Frage des politischen Willens, nationalstaatliche Rahmenbedingungen so zu gestalten, dass ein an Menschen- und Kinderrechten orientiertes professionelles Handeln im Kontext von Flucht und Asyl möglich ist. Nicht selten stehen nationalstaatliche Interessen einer Gleichbehandlung von Menschen mit und ohne Staatsbürger_innenschaft des jeweiligen Landes entgegen und die Soziale Arbeit droht, primär Exklusionsverwalterin anstatt Exklusionsvermeiderin und Inklusionsvermittlerin zu sein (Bommes und Scherr 1996). Sie ist eingebettet in ein „Spannungsverhältnis zwischen normativ begründbaren moralischen Konzepten einerseits, politisch und/oder ökonomisch definierten Interessenlagen anderseits“ (Scherr 2020, S. 137). Volle Bürger_innenrechte werden vor allem „einer exklusiven Wir-Gruppe“ (Mecheril 2020, S. 102) gewährt, während Rechtsansprüche für geflüchtete Menschen ohne deutsche Staatsbürgerschaft begrenzt bleiben (Goebel 2018, S. 91). Dabei formuliert die Allgemeine Erklärung der Menschenrechte in Artikel 28: „Jeder Mensch hat Anspruch auf eine soziale und internationale Ordnung, in welcher die in der vorliegenden Erklärung angeführten Rechte und Freiheiten voll verwirklicht werden können“. Hierdurch wird die „menschenrechtsangemessene Gestaltung von Gesellschaften " (Scherr 2011, S. 10) als Querschnitts- und bisher nur unzureichend vollzogene globale Aufgabe in den Blick gerückt. Diese sei, so Scherr (2020, S. 138), ausschließlich als „Utopie einer Weltgesellschaft" mit überall verwirklichten Menschenrechten denkbar.

\section{Neue Wege explorieren}

Das Spannungsfeld von universalistischem Gleichheitsanspruch und der Durchsetzung nationalstaatlicher Partikularinteressen führt in der wissenschaftlichen Debatte zu verschiedenen Positionen: während die einen transnationale Selbstbestimmungs- und Bewegungsrechte (Mecheril 2020) als zukunftsweisende Wege reflektieren, gehen andere von der Unauflösbarkeit dieser Konfliktlinien unter „Bedingungen der realen Macht- und Ungleichheitsverhältnisse [aus] “ (Scherr 2020, S. 138). Wie zentral ein Weiterführen und Aushandeln dieser Debatten ist, zeigt indes die Empirie: Die Fallvignette, die exemplarisch das spannungsvolle Agieren einer Pädagogin im Kontext von Flucht und Asyl herausstellt, verlangt geradezu nach neuen Überlegungen zur Anerkennung transnationaler Lebenswelten, zu postnationalen Gesellschaftskonzepten (Goebel 2018, S. 94) und nach Übersetzer_innen (Staub-Bernasconi 2019, S. 173), die sich für eine Umsetzung und Einhaltung von Menschenrechten in ihrer inter- und nationalstaatlichen Rückbindung einsetzen - in Protesten, Solidaritätsprojekten und im pädagogischen Handeln. Dabei scheint uns zentral, über die bestehenden Verhältnisse hinauszudenken und neue Wege zu explorieren, wie sie vor allem in zivilgesellschaftlichen Allianzen entwickelt werden. Zentrale Aufgabe ist die Schaffung neuer Instanzen, etwa einer flächendeckenden Verankerung unabhängiger Ombudsstellen auch im Feld von Fluchtmigration, die Pädagog_innen wie auch geflüchteten Menschen in den Unterkünften Ansprechpartnerin sind, das Menschenrechtsschutzsystem der Vereinten Nationen nutzen und von Beschwerde- und Meldeverfahren Gebrauch machen (Prasad 2018, S. 22). Diese Überlegungen werden getragen von der grundlegenden Frage danach, wie die Soziale Arbeit zu einem würdevollen Leben für alle Menschen - unabhängig von dem Kriterium einer spezifischen Staatsangehörigkeit - in einer von sozialen Ungleichheiten durchzogenen Welt beitragen kann. Die Breite der Debatte erachten wir in diesem Zusammenhang als wertvoll. Sie zeigt - trotz und wegen aller Herausforderungen - wie zentral eine menschenrechtsbasierte Fundierung Sozialer Arbeit ist, die 
ihren Anspruch der schwierigen Suche nach Inklusion in einer transnationalisierten Gesellschaft nicht aufgeben darf.

Eingegangen. 3. August 2020

Angenommen. 8. September 2020

Funding. Open Access funding enabled and organized by Projekt DEAL.

Open Access. Dieser Artikel wird unter der Creative Commons Namensnennung 4.0 International Lizenz veröffentlicht, welche die Nutzung, Vervielfältigung, Bearbeitung, Verbreitung und Wiedergabe in jeglichem Medium und Format erlaubt, sofern Sie den/die ursprünglichen Autor(en) und die Quelle ordnungsgemäß nennen, einen Link zur Creative Commons Lizenz beifügen und angeben, $o b$ Änderungen vorgenommen wurden.

Die in diesem Artikel enthaltenen Bilder und sonstiges Drittmaterial unterliegen ebenfalls der genannten Creative Commons Lizenz, sofern sich aus der Abbildungslegende nichts anderes ergibt. Sofern das betreffende Material nicht unter der genannten Creative Commons Lizenz steht und die betreffende Handlung nicht nach gesetzlichen Vorschriften erlaubt ist, ist für die oben aufgeführten Weiterverwendungen des Materials die Einwilligung des jeweiligen Rechteinhabers einzuholen.

Weitere Details zur Lizenz entnehmen Sie bitte der Lizenzinformation auf http://creativecommons.org/licenses/by/4.0/deed.de

1. Die Materialerhebung ist in ein Forschungsprojekt zum Thema „Sozialraum „AnkER-Zentrum“. Lebenswirklichkeiten geflüchteter Menschen in Deutschland" eingebettet, das von Anett Schmitz (Ethnologie, Universität Trier) und Caroline Schmitt (Sozialpädagogik, Johannes Gutenberg-Universität Mainz) seit Juni 2019 durchgeführt wird.

2. Der Name wurde durch ein Pseudonym ersetzt.

\section{Literatur}

Bauer, I. (2017). Unterbringung von Flüchtlingen in deutschen Kommunen. State-of-Research Papier 10. https://flucht-forschung-transfer.de/wpcontent/uploads/2017/05/IB-SoR-10-BAUER_Konfliktmediation-1.pdf. Zugegriffen: 30. Juli 2020.

Bayerischer Flüchtlingsrat (2019). Schriftliche Stellungnahme zur Expert"innenanhörung zum Thema „AnkER-Einrichtungen in Bayern“. https://www.fluechtlingsrat-bayern.de/tl_files/2019/Dokumente/Statement\%20Frau\%20Grote.pdf. Zugegriffen: 30. Juli 2020.

Bommes, M., \& Scherr, A. (1996). Soziale Arbeit als Exklusionsvermeidung, Inklusionsvermittlung und/oder Exklusionsverwaltung. In R. Merten, P. Sommerfeld \& T. Koditek (Hrsg.), Sozialarbeitswissenschaft - Kontroversen und Perspektiven (S. 93-119). Neuwied: Luchterhand.

Bundesministerium für Familie, Senioren, Frauen und Jugend (BMFSFJ), \& United Nations Children's Fund (UNICEF) (2018). Mindeststandards zum Schutz von geflüchteten Menschen in Flüchtlingsunterkünften. Berlin. https://www.gewaltschutz-gu.de/publikationen/mindeststandards/ download/mindeststandards-zum-schutz-von-gefluechteten-menschen-influechtlingsunterkuenften-bmfsfj-unicef-u-a-3-aufl-2018. Zugegriffen: 8 . Sept. 2020.

Bundeskriminalamt (2019). Kriminalität im Kontext von Zuwanderung. Kernaussagen Betrachtungszeitraum: 01.01.-30.09.2019. Wiesbaden: BKA.
Eichinger, U., \& Schäuble, B. (2018). Gestalten unter unmöglichen Bedingungen? Soziale Arbeit in Gemeinschaftsunterkünften. In Nivedita Prasad (Hrsg.), Soziale Arbeit mit Geflüchteten. Rassismuskritisch, professionell, menschenrechtsorientiert (S. 274-299). Opladen, Toronto: Barbara Budrich.

Furman, et al. (2008). Transnational social work: using a wraparound model. Global Networks, 8(4), 496-503.

Goebel, S. (2018). Menschenrechte und Internationale Soziale Arbeit in transnationalen Gesellschaften. In B. Blank \& al (Hrsg.), Soziale Arbeit in der Migrationsgesellschaft (S. 87-96). Wiesbaden: VS.

Healy, L. M. (2008). International social work. Professional action in an interdependent world (2. Aufl.). Oxford: Oxford University Press.

Kleibl, T., \& Lutz, R. (2020). Globale Ungleichheiten. Herausforderungen für eine internationale Soziale Arbeit. In C. Steckelberg \& B. Thiessen (Hrsg.), Wandel der Arbeitsgesellschaft (S. 51-65). Opladen, Berlin, Toronto: Budrich.

Mecheril, P. (2020). Gibt es ein transnationales Selbstbestimmungsrecht? In I. van Ackeren, et al. (Hrsg.), Beiträge zum 26. Kongress der Deutschen Gesellschaft für Erziehungswissenschaft (S. 101-117). Opladen: Budrich.

Prasad, N. (2018). Statt einer Einführung: Menschenrechtsbasierte, professionelle und rassismuskritische Soziale Arbeit mit Geflüchteten. In N. Prasad (Hrsg.), Soziale Arbeit mit Geflüchteten (S. 9-29). Opladen, Toronto: Budrich.

Röh, D. (2013). Die sozialen Grundlagen der Menschenrechte - transforming rights into capabilities. In E. Mührel \& B. Birgmeier (Hrsg.), Menschenrechte und Demokratie (S. 143-161). Wiesbaden: VS.

Schäuble, B. (2018). Sozialarbeiter*innen als „social workers without borders“" oder als „borderworker"? In Bundesweite Arbeitsgemeinschaft der Psychosozialen Zentren für Flüchtlinge und Folteropfer (Hrsg.), Von Aleppo nach Eisenhüttenstadt - und dann? Tagungsdokumentation, Berlin. (S. 89-94).

Scherr, A. (2020). Grenzziehungen: Soziologie der paradoxen Forderung nach offenen Grenzen. In D. Kersting \& M. Leuoth (Hrsg.), Der Begriff des Flüchtlings (S. 137-155). Berlin: Springer VS.

Scherr, A. (2011). Menschenrechte als gesellschaftspolitische Orientierung? In Komitee für Grundrechte \& D. V. e (Hrsg.), Der Kampf um Menschenrechte im Zeitalter kapitalistisch entfesselter Globalisierung. Tagungsdokumentation, Köln. (S. 10-16).

Schiffauer, W., Eilert, A., \& Rudloff, M. (2017). So schaffen wir das - eine Zivilgesellschaft im Aufbruch. 90 wegweisende Projekte mit Geflüchteten. Bielefeld: transcript.

Staub-Bernasconi, S. (2019). Menschenwürde - Menschenrechte - Soziale Arbeit. Opladen, Berlin, Toronto: Budrich. 
Hier steht eine Anzeige.

\section{Springer}

